\title{
Les comités d'éthique clinique en Suisse - un état des lieux
}

\author{
M. Salathé, M. Leuthold, H. Amstad, M. Vallotton
}

Deutsch erschienen

in Nr. 43/2003.

1 Rüetschi D. Ärztliches Standesrecht in der Schweiz - Die Bedeutung der medizinischethischen Richtlinien der Schweizerischen Akademie der Medizinischen Wissenschaften. Jahrbuch Junger Zivilrechtswissenschaftler 2002: Die Privatisierung des Privatrechts - rechtliche Gestaltung ohne staatlichen Zwang. Stuttgart: Richard Boorberg Verlag; 2002.

2 Les recommandations de la Société Suisse de Néonatologie concernant la prise en charge des prématurés à la limite de la viabilité.

3 Mc Gee G, Caplan AL, Spagnola JP, Asch DA. A national study of ethics committees. Am J Bioeth 2001;1(4):60-4

4 Chevrolet J-C. Les comités locaux d'éthique clinique hospitaliers. Med Hyg 2002;60:1781-6.

5 Kettner M, May A. EthikKomitees in Kliniken Bestandesaufnahme und Zukunftsperspektive. Ethik Med 2002;14:295-7.

Correspondance:

Michelle Salathé, lic. iur.

ASSM

Petersplatz 13

CH-4051 Bâle

\section{Résumé}

Selon un sondage réalisé auprès d'hôpitaux et d'institutions suisses, les questions et conflits éthiques rencontrés dans la pratique clinique quotidienne concernent le plus souvent les décisions liées à une éventuelle interruption du traitement $(47 \%)$, suivis des conflits avec les proches (42\%), de l'alimentation artificielle (39\%), des indications pour des interventions chirurgicales (38\%), des mesures de contrainte (31\%), des conflits avec des patients (30\%), de la réanimation $(26 \%)$ et de l'assistance au décès (20\%).

Les établissements suisses ne bénéficient que trop rarement du soutien de comités d'éthique clinique dans la gestion de tels conflits. Seulement $20 \%$ des hôpitaux et institutions suisses disposent d'un comité d'éthique. Ceux-ci se trouvent dans des hôpitaux pour soins aigus, plus rarement dans des institutions psychiatriques et à peine dans des institutions de soins. Aucune clinique de réadaptation ne dispose d'un comité d'éthique.

Ces comités assument, d'une part, un rôle de conseiller de la direction de l'établissement (88\%) ainsi qu'une fonction consultative dans les cas particuliers (84\%) voire même une compétence décisionnelle (31\%); d'autre part, ils sont chargés de l'élaboration de directives internes concernant des questions médicoéthiques (61\%). Tous les comités d'éthique ont une composition interdisciplinaire.

\section{Contexte}

Dans leur activité clinique quotidienne, médecins et soignants sont régulièrement confrontés à des situations qui appellent des décisions graves et délicates sur le plan éthique: naissance de grands prématurés ou d'enfants gravement handicapés, prescriptions de non-réanimation, débranchement d'un cœur-poumon artificiel ou d'un respirateur, etc. Les directives médicoéthiques, telles que les publient par exemple l'Académie Suisse des Sciences Médicales (ASSM) ou la Deutsche Bundesärztekammer (BÄK), ont pour but d'aider les intervenants à faire face à de telles situations. Celles-ci fournissent aux médecins praticiens confrontés à des situations difficiles un appui pour agir de la façon la plus adéquate possible et prendre la meilleure décision (pour des informations détaillées à ce sujet: [1]). Les directives des sociétés de disciplines médicales (p.ex. [2]) vont dans le même sens.

Toutefois, des directives ne peuvent être que des aides. La décision en elle-même doit être prise en fonction du cas particulier et de ses spécificités. A cet égard, les comités d'éthique clinique, mais aussi les discussions éthiques internes ou la consultation d'un spécialiste en éthique, peuvent se révéler précieux. Les comités d'éthique clinique sont définis comme étant des instances interdisciplinaires - consultatives ou décisionnelles - traitant au cas par cas de problématiques médico-éthiques. Ils peuvent être permanents ou constitués uniquement lorsque le besoin s'en fait sentir.

Aux Etats-Unis, les comités d'éthique clinique existent depuis les années 70 [3]. En Europe, ils ont fait leur apparition beaucoup plus tardivement [4]. En Allemagne, par exemple, ce n'est qu'en 1997 qu'une septantaine d'établissements confessionnels se sont dotés d'un comité d'éthique, sur recommandation de la fédération des hôpitaux catholiques et protestants. S'agissant des établissements non confessionnels, les comités d'éthique sont actuellement au nombre d'une demi-douzaine [5]. Il n'existe pour l'instant de recensement systématique des comités d'éthique clinique dans aucun autre pays européen. Ni en Suisse d'ailleurs. C'est pour cette raison que la Commission centrale d'éthique (CCE) de l'ASSM a mené l'année dernière une enquête dans ce domaine. Les buts visés étaient les suivants:

- avoir une idée de la fréquence des situations qui, dans l'activité clinique quotidienne, entraînent des conflits médico-éthiques (selon le type de conflit et le genre d'établissement); - recenser les comités d'éthique clinique existant dans les hôpitaux et homes médicalisés suisses;

- cerner les tâches et la structure de ces comités;

- évaluer le besoin de mise en réseau de ces comités. 
Tableau 1

Fréquence des différents types de conflits en fonction du genre d'établissement.

\begin{tabular}{lllllll}
\multicolumn{2}{l}{ Hôpital pour soins aigus } & \multicolumn{4}{l}{ Etablissement psychiatrique } & \multicolumn{2}{l}{ Home médicalisé } \\
Arrêt du traitement & $60 \%$ & Mesures de contrainte & $88 \%$ & Indication opératoire & $68 \%$ \\
\hline Réanimation & $41 \%$ & Conflits avec des patients & $65 \%$ & Conflits avec des proches & $66 \%$ \\
\hline Indication opératoire & $34 \%$ & Conflits avec des proches & $31 \%$ & Alimentation artificielle & $58 \%$ \\
\hline Euthanasie & $34 \%$ & Alimentation artificielle & $31 \%$ & Arrêt du traitement & $47 \%$ \\
\hline Conflits avec des proches & $32 \%$ & Arrêt du traitement & $27 \%$ & Mesures de contrainte & $37 \%$ \\
\hline Alimentation artificielle & $28 \%$ & Indication opératoire & $15 \%$ & Conflits avec des patients & $26 \%$
\end{tabular}

\section{Méthode}

Dans le cadre d'une enquête par questionnaire, il a été demandé à tous les hôpitaux et homes médicalisés affiliés à la fédération des hôpitaux $\mathrm{H}+$ (état en janvier 2002) d'indiquer s'ils disposaient d'un comité d'éthique clinique. Tous les établissements qui ont répondu à ce premier questionnaire ont reçu par la suite un second questionnaire, plus détaillé avec des questions concernant le type et l'importance de l'hôpital ainsi que les conflits éthiques survenant le plus fréquemment dans la pratique clinique quotidienne. A cet égard, onze domaines tels que les interruptions de grossesses à un stade avancé, les mesures de contrainte, etc. étaient énumérés, avec la possibilité d'en indiquer plusieurs à la fois mais également de compléter la liste. D'autres questions concernaient la gestion des conflits (procédures standardisées, type de prise de décision, etc.). Pour les institutions qui avaient indiqué qu'elles disposent d'un comité d'éthique, le questionnaire comportait des questions sur ces comités (fonctions, constitution, etc.).

\section{Résultats}

\section{Fréquence / Retour}

A l'issue du premier questionnaire, envoyé à 393 établissements, 57 (18\%) ont indiqué disposer d'un comité d'éthique et 261 (82\%) ont déclaré ne pas en disposer. Le taux de retour était de $81 \%$.

Le taux de retour du second questionnaire détaillé, qui n'a été envoyé qu'aux 318 établissements ayant répondu au premier questionnaire, était de $61 \%$. Sur les 57 institutions qui, à l'issue $\mathrm{du}$ premier questionnaire, avaient indiqué disposer d'un comité d'éthique clinique, 26 d'entre elles (46\%) ont répondu à ce second questionnaire. Sur les 261 hôpitaux et homes médicalisés ne disposant pas d'un comité d'éthique clinique, $172(66 \%)$ ont répondu à ce second questionnaire.

\section{Conflits médico-éthiques survenant dans I'activité clinique quotidienne}

La totalité des établissements (qu'ils aient ou non un comité d'éthique clinique) ont été interrogés sur les conflits survenant le plus fréquemment dans l'activité clinique quotidienne. Au premier rang de ces conflits figurent les décisions difficiles liées à une éventuelle interruption du traitement $(47 \%)$, suivies des conflits avec des proches (42\%). Puis viennent l'alimentation artificielle $(39 \%)$, les indications pour des interventions chirurgicales (38\%), les mesures de contrainte $(31 \%)$, les conflits avec des patients $(30 \%)$, la réanimation $(26 \%)$ et l'euthanasie (20\%). Les questions et conflits éthiques dans les domaines du rationnement (10\%), de l'interruption tardive de grossesse $(6 \%)$ et de la transplantation (3\%) ont été beaucoup plus rarement cités. Comme le montre le tableau ci-dessus, la fréquence de ces conflits varie selon le genre d'établissement.

\section{Gestion des conflits médico-éthiques surve- nant dans l'activité clinique quotidienne}

Dans 61\% des institutions ayant participé au sondage, la possibilité existe de recourir à des procédures spéciales telles que par exemple la mise sur pied d'un conseil d'éthique*, ou encore la consultation d'un éthicien (service consultatif en éthique) ${ }^{\star *}$, cela en plus des comités d'éthique clinique éventuellement existants. 94\% des établissements ont déclaré que, dans des situations délicates du point de vue éthique, une décision prise en équipe est dans toute la mesure du possible recherchée, les membres de l'équipe étant alors, dans 53\% des cas, désignés par le médecinchef responsable. Sur les 57 institutions qui ont indiqué disposer d'un comité d'éthique clinique, près de la moitié (46\%) ont déclaré, lors du second questionnaire, faire appel au comité d'éthique clinique dans de telles situations.

\footnotetext{
** Un conseil d'éthique assume alors la tâche clinique de débattre du cas concret.

* Par conseil d'éthique, on entend sein de l'équipe soignante, discussion approfondie des question thiques qui se posent.
} 


\section{Diffusion des comités d'éthique clinique}

$19 \%$ des hôpitaux pour soins aigus, 15\% des cliniques psychiatriques et $3 \%$ des institutions de soins disposent de comités d'éthique clinique. Ils sont en revanche inexistants dans les cliniques de réadaptation. Le comité d'éthique le plus «ancien» date de 1988, et tout juste la moitié des comités d'éthique existent depuis 1 à 2 ans.

\section{Composition et taille des comités d'éthique clinique}

Tous les comités d'éthique ont une composition interdisciplinaire. Les médecins y sont toujours représentés, ainsi que, le plus souvent, le personnel soignant $(85 \%)$. Souvent, des juristes (56\%), théologiens (52\%) et éthiciens (46\%) y siègent également. On trouve beaucoup plus rarement dans ces comités des représentants de l'administration (36\%) et des psychologues (28\%). La taille des comités varie de 3 (au minimum) à 18 membres, la plupart des comités d'éthique comportant de 6 à 9 membres. Des intervenants externes siègent dans $87 \%$ des comités d'éthique.

\section{Tâches des comités d'éthique clinique}

Presque tous les comités d'éthique conseillent la direction de l'établissement (88\%) et ont une fonction consultative dans les cas particuliers (84\%). 30\% ont indiqué avoir aussi une compétente décisionnelle dans les cas particuliers. La plupart de ces comités sont également responsables de l'élaboration de directives internes (61\%).

Le plus souvent, le recours aux comités d'éthique clinique se fait sur une base volontaire; $27 \%$ seulement des établissements connaissent une obligation de faire appel au comité d'éthique clinique en face d'une décision délicate à prendre. En cas de recours volontaire au comité d'éthique, ce dernier peut généralement être actionné par tous les intervenants: médecinschefs, médecins assistants et chefs de clinique toujours, soignants souvent (70\%), mais aussi personnes concernées (patient et proches).

\section{Fonctionnement des comités d'éthique clinique}

La plupart des comités d'éthique clinique travaillent sur la base d'un règlement écrit définissant leurs tâches, leur composition. Il existe toutefois de grandes différences en ce qui concerne le degré de détail de ces dispositions (nomination des membres, durée de leur mandat, règles de récusation, obligation de faire figurer les décisions dans un procès-verbal, etc.). Il existe en outre des différences dans la fréquence des séances (convocation d'une séance sur demande en l'espace de quelques heures; séances régulières fixées à l'avance). En règle générale, les décisions des comités d'éthique doivent être adoptées à l'unanimité; toutefois, $27 \%$ des comités d'éthique ont indiqué qu'ils peuvent également faire valoir une décision majoritaire à défaut de consensus.

$37 \%$ des comités d'éthique entretiennent un échange avec d'autres comités d'éthique. Une proportion beaucoup plus élevée (75\%) des comités d'éthique appellent de leurs vœux une mise en réseau.

\section{Conclusions}

Les principaux types de conflits correspondent à l'idée qu'on pouvait en avoir: l'arrêt d'un traitement, les indications pour des opérations chirurgicales, l'alimentation artificielle, mais aussi les mesures de contrainte - tout particulièrement en psychiatrie et dans les homes médicalisés sont des aspects extrêmement sensibles. Il n'est pas rare qu'il s'agisse d'une question de vie ou de mort, ce qui requiert un processus décisionnel minutieux associant de la même manière les personnes concernées et l'équipe soignante.

Bien que les sources de conflits qui viennent d'être citées soient fréquemment liées à la prise en charge de patients âgés et dépendants, les comités d'éthique clinique sont très rares dans les homes médicalisés. Les personnes âgées en situation de dépendance, qui n'ont souvent plus de famille, sont pourtant tributaires d'une aide particulièrement attentive dans les situations où des décisions difficiles sont à prendre.

A peine vingt pour cent des hôpitaux et homes médicalisés suisses ont institué des comités d'éthique clinique. Mais il existe le plus souvent d'autres structures venant appuyer la prise de décisions médico-éthiques dans les cas individuels (services consultatifs par ex.). Toutefois, elles n'ont pas encore été recensées de façon systématique.

Les comités d'éthique clinique remplissent généralement plusieurs missions: sensibiliser le personnel aux questions éthiques dans l'activité clinique quotidienne, favoriser la capacité à émettre un jugement éthique et formuler des directives générales pour l'activité clinique dans la gestion des questions éthiques, mais aussi et surtout ils soutiennent et déchargent le personnel (ainsi que les patients et leurs proches) en participant à la prise de décision. En offrant des garanties de procédure en matière de transparence de la prise de décision, ils contribuent à l'accep- 
6 Levine C. Questions and (some very tentative) answers about hospital ethics committees. Hastings Cent Rep 1984; 14(3):9-12. tation des décisions. En outre, grâce à leur expérience et leur continuité, ils peuvent aussi tirer des conclusions pour l'avenir sur la base de l'analyse rétrospective des cas traités.

Un tiers des comités d'éthique clinique en Suisse ont une compétence décisionnelle dans les cas particuliers, mais on peut supposer de façon générale que les comités d'éthique sont souvent décisionnaires de facto même lorsqu'ils n'ont pas de compétence formelle de décision. Dans la littérature, américaine en particulier, cet état de fait est critiqué [6]. Les comités d'éthique clinique ne devraient pas être les décisionnaires primaires et, surtout, ne devraient pas se substituer aux instances juridiques ou, le cas échéant, judiciaires. Il semblerait qu'en Suisse un tiers des comités d'éthique clinique a une compétence décisionnelle dans les cas particuliers; dès lors il est nécessaire de clarifier la question de la responsabilité juridique de l'exécution des décisions. A cet égard, il est important de connaître les bases légales des comités d'éthique, de savoir par qui elles ont été instaurées (autorité, direction de clinique) et si leur consultation est obligatoire ou non. A l'inverse, même lorsqu'un comité d'éthique clinique n'a qu'une fonction consul- tative, les médecins responsables devraient prendre en compte son avis défavorable concernant l'exécution ou l'interruption d'une mesure, pour éviter de faire l'objet de reproches par la suite.

L'enquête de l'ASSM a mis en évidence un gros besoin en matière d'échange d'informations entre les comités d'éthique. «Dialog Ethik» à Zurich organise déjà des réunions régulières entre les forums d'éthique clinique qu'il anime, forums qui se limitent toutefois pour l'essentiel à l'agglomération zurichoise. En tant qu'organisation active à l'échelle nationale, l'ASSM s'emploiera à favoriser davantage cet échange. C'est pourquoi, elle prévoit le 30 avril 2004 une rencontre de tous les comités d'éthique suisses (cf. cadre ci-dessous) et proposera un forum pour la présentation des différents modèles de «conseil en éthique» et des expériences faites dans ce cadre, ainsi que pour un échange général d'expériences entre les comités d'éthique clinique.

Nous tenons à remercier de leur obligeance tous les établissements qui ont pris la peine de remplir nos questionnaires et de nous les retourner.

\section{Vorankündigung}

Informations- und Erfahrungsaustausch für klinische Ethikkommissionen: Tagung der SAMW am 30. April 2004

Die Umfrage der SAMW bei den klinischen Ethikkommissionen hat gezeigt, dass ein grosses Bedürfnis nach einem Informations- und Erfahrungsaustausch besteht. Aus diesem Grund organisiert die SAMW am 30. April 2004 eine Tagung, die sich an Mitglieder von klinischen Ethikkommissionen und -foren, interessierte Ärztinnen und Ärzte sowie Spital- und Pflegeheimleitungen richtet.

Im Rahmen dieser Veranstaltung werden verschiedene Modelle vorgestellt, wie im klinischen Alltag mit ethisch heiklen Fragestellungen umgegangen werden kann. Selbstverständlich steht auch genügend Zeit für den Erfahrungsaustausch, für die Beantwortung von Fragen und für die Kontaktpflege zur Verfügung.

Das definitive Programm erscheint im Februar 2004.

\section{Préavis}

Echange d'informations et d'expérience entre les différents comités d'éthique clinique: colloque de I'ASSM le 30 avril 2004

L'enquête de l'ASSM auprès des comités d'éthique clinique a mis en évidence un gros besoin en matière d'échange d'informations. C'est pourquoi, l'ASSM organise une journée de réflexion pour les membres de comités et de forums d'éthique clinique, les médecins intéressés ainsi que les directions d'hôpitaux et d'institutions de soins.

Dans le cadre de cette manifestation seront présentées différentes façons de gérer les problèmes éthiques délicats rencontrés dans la pratique clinique quotidienne. Un temps suffisant sera réservé pour l'échange d'expériences, les questions ouvertes et les contacts entre les membres des différents comités d'éthique.

Le programme définitif sera publié en février 2004. 